\title{
Cinética de espermatozoides criopreservados de bovinos após sexagem por citometria de fluxo
}

\author{
José de Oliveira Carvalho( ${ }^{(1)}$, Roberto Sartori(2), Amanda Prudêncio Lemes ${ }^{(3)}$, Gerson Barreto Mourão(2) \\ e Margot Alves Nunes Dode ${ }^{(4)}$
}

\begin{abstract}
(1)Universidade de Brasília, Faculdade de Agronomia e Medicina Veterinária, Campus Universitário Darcy Ribeiro, Caixa Postal 04561, CEP 70910-900 Brasília, DF. E-mail: joseocneto@hotmail.com (2)Universidade de São Paulo, Escola Superior de Agricultura Luiz de Queiroz, Caixa Postal 09, CEP 13418-900 Piracicaba, SP. E-mail: sartori@esalq.usp.br, gbmourão@esalq.usp.br (3)Universidade para o Desenvolvimento do Estado e da Região do Pantanal, Caixa Postal 2153, CEP 79003-010 Campo Grande, MS. E-mail: amanda-lemes@hotmail.com ${ }^{(4)}$ Embrapa Recursos Genéticos e Biotecnologia, Caixa Postal 02372, CEP 70770-917 Brasília, DF. E-mail: margot@cenargen.embrapa.br
\end{abstract}

Resumo - O objetivo deste estudo foi comparar a cinética de sêmen bovino criopreservado não sexado, sexado $\mathrm{X}$ e sexado $\mathrm{Y}$ antes e depois da seleção espermática por gradiente de Percoll. Amostras criopreservadas de sêmen não sexado (grupo NS) e sexado X (grupo SX) e Y (grupo SY) por citometria de fluxo, de quatro touros, foram avaliadas quanto à motilidade e à cinética espermática com o "computer-assisted semen analysis" (CASA) e o restante da amostra de cada grupo foi submetido à seleção espermática em gradiente de Percoll (45:60\%). Após a seleção, foram realizadas as mesmas avaliações que antes da passagem pelo Percoll. A motilidade do grupo NS foi superior à dos grupos SX e SY e não foi observada diferença entre os grupos SX e SY nos parâmetros de cinética espermática obtidos pelo CASA, antes ou após a passagem pelo Percoll. Foi observado aumento na motilidade para todos os grupos como efeito da seleção pelo Percoll. O processo de sexagem por citometria de fluxo afeta a cinética espermática, e a passagem pelo Percoll aumenta a motilidade do sêmen sexado e não sexado sem alterar a cinética do sêmen não sexado.

Termos para indexação: gradiente de Percoll, motilidade espermática, sêmen sexado.

\section{Kinetics of bovine cryopreserved sperm cells after sexing by flow cytometry}

\begin{abstract}
The objective of this study was to compare the kinetics of bovine cryopreserved nonsexed, sexed for $\mathrm{X}$ and sexed for $\mathrm{Y}$ sperm cells before and after selection using Percoll gradient. Cryopreserved nonsexed (NS group) semen samples and sexed for X (SX group) and for Y (SY group) by flow cytometry, from four different sires, were analyzed for motility by computer-assisted semen analysis (CASA), and the remainder of the sperm samples for each group was submitted to Percoll (45:60\%) gradient selection. After Percoll selection, the same sperm analyses done before the passing through Percoll gradient were performed. Sperm motility was higher in the NS group than in the SX and SY groups, and no differences were observed between these last two groups for any of the sperm parameters evaluated by CASA, either before or after Percoll selection. Percoll increased motility for all groups. Changes in other characteristics evaluated by CASA were observed only in the NS group. Sex-sorting procedure by flow cytometry affected sperm kinetics, and Percoll gradient selection increases motility in sexed and nonsexed sperm without affecting the kinetics of nonsexed sperm.
\end{abstract}

Index terms: Percoll gradient, sperm motility, sexed sperm.

\section{Introdução}

Nas últimas décadas, várias tecnologias foram desenvolvidas na área de reprodução animal. Uma das biotécnicas recentes é a sexagem espermática por citometria de fluxo, que permite a separação de espermatozoides de acordo com o cromossomo $\mathrm{X}$ ou Y. Esse método baseia-se na diferença do conteúdo de DNA dos espermatozoides, pois, em bovinos, o cromossomo X contém aproximadamente $4 \%$ a mais de DNA do que o cromossomo Y (Almeida \& Alvarez, 2003).

A utilização comercial do sêmen sexado na reprodução de bovinos é uma realidade, apesar de muitos estudos relatarem menor fertilidade do mesmo quando comparado ao não sexado, tanto in vivo como in vitro (Sartori et al., 2004; Bodmer et al., 2005; Anderson et al., 2006; Bermejo-Álvarez et al., 2008). A técnica de sexagem por citometria de fluxo pode gerar alguns danos aos espermatozoides, provavelmente em razão 
da exposição ao laser, da grande pressão na passagem pelo citômetro, da queda em grande velocidade dentro do tubo de colheita e da permanência durante algumas horas em temperatura ambiente antes do processamento (Chastant-Maillard \& Druat, 2005).

Entre as características espermáticas afetadas pelo processo de sexagem, a diminuição da motilidade tem sido relatada por diversos autores (Hollinshead et al., 2004; Blondin et al., 2009). De acordo com Fonseca et al. (1992), a motilidade é um importante fator a ser considerado na análise da qualidade espermática, e constitui um dos melhores parâmetros para se predizer a fertilidade de uma amostra de sêmen (Varner et al., 1991; Malmgren, 1997; Januskauskas et al., 2000; Suarez \& Pacey, 2006).

O método mais utilizado para avaliação da motilidade é a determinação subjetiva da percentagem de células móveis em uma amostra de sêmen com uso de microscopia óptica. Esse método é uma forma indireta de avaliação, simples e de baixo custo, no entanto pode apresentar grande variabilidade (Malmgren, 1997; Verstegen et al., 2002). Na busca de maior objetividade nessa avaliação, vários métodos têm sido propostos, entre eles o sistema de análise computadorizada do movimento espermático - "computer-assisted semen analysis" (CASA). Esse tipo de análise permite uma avaliação mais exata e objetiva da motilidade, fornece informações precisas e significativas da cinética da célula espermática (Mortimer, 1997; Cox et al., 2006) e determina não somente a percentagem de células móveis na amostra, mas também quantifica características específicas do movimento espermático (Garner, 1997). No entanto, para avaliação de motilidade, não há, na literatura, trabalho com utilização de sêmen sexado X, sexado $\mathrm{Y}$ e não sexado de um mesmo ejaculado, e isso é um importante ponto a ser considerado, já que foi demonstrado que diferentes ejaculados podem gerar amostras de sêmen com diferentes características (Blondin at al., 2009). Dessa forma, o objetivo do presente estudo foi comparar variáveis da cinética de sêmen bovino criopreservado não sexado, sexado $\mathrm{X}$ e sexado Y pelo sistema de análise CASA antes e após a seleção espermática por gradiente de Percoll.

\section{Material e Métodos}

O experimento foi realizado no Laboratório de Reprodução Animal da Embrapa Recursos Genéticos e Biotecnologia, em Brasília, DF, entre os meses de janeiro e abril de 2009.

Amostras de sêmen congelado de quatro touros da raça Nelore, mantidos em central de inseminação artificial, foram utilizadas no presente estudo. Para a produção do material experimental, um ejaculado de cada touro foi coletado e fracionado em três partes. Uma parte foi separada para constituir o grupo não sexado (grupo NS) e as outras duas foram submetidas à citometria de fluxo, para produção de espermatozoides com cromossomo X (grupo SX) ou Y (grupo SY). Após a composição dos grupos, todas as amostras foram congeladas. Todo o procedimento foi realizado por empresa comercial especialista na área (ABS Pecplan, Uberaba, MG), e as amostras foram adquiridas dessa empresa. O sêmen foi, então, utilizado para avaliação da cinética espermática, e cada procedimento foi repetido três vezes. Em cada repetição, uma palheta da mesma partida de cada grupo (NS, SX e SY) foi descongelada a $37^{\circ} \mathrm{C}$ por 30 segundos em banho-maria, e uma amostra foi retirada para avaliação subjetiva da motilidade e avaliação da cinética espermática pelo sistema computadorizado CASA (modelo Ivos-Ultimate 12, Hamilton Thorne Biosciences, França).

Para avaliação subjetiva, uma gota de sêmen foi colocada sobre uma lâmina aquecida e coberta com lamínula, e analisada em microscópio de contraste de fase com aumento de 20x. Para a avaliação da motilidade pelo CASA, $2 \mu \mathrm{L}$ de sêmen foram colocados na lâmina de leitura (Leja standard count, SC20.01.04.B, $20 \mu \mathrm{m}$, Leja Product B.V., Holanda) e a amostra foi avaliada no aparelho previamente ajustado, conforme as recomendações do fabricante para análise de sêmen bovino. No mínimo sete campos foram selecionados manualmente para a leitura e análise. Os parâmetros de cinética espermática mensurados foram: motilidade total (MT), em percentagem; velocidade de trajeto (VAP), em micrômetro por segundo; velocidade retilínea (VSL), em micrômetro por segundo; velocidade curvilínea (VCL), em micrômetro por segundo; amplitude lateral de cabeça (ALH), em micrômetro; frequência de batimentos (BCF), em Hz; e linearidade (LIN), em percentagem.

O restante da amostra de cada grupo foi utilizado em protocolo de seleção espermática para sêmen sexado, de acordo com Blondin et al. (2009), com modificações. Para isso, as amostras de sêmen foram colocadas em gradiente de Percoll (GE Healthcare, 
Upsalla, Suécia), constituído de $400 \mu \mathrm{L}$ de Percoll $45 \%$ e $400 \mu \mathrm{L}$ de Percoll $60 \%$, e centrifugadas a $700 \mathrm{~g}$ por 5 min. Após centrifugação, o sobrenadante foi descartado e o pellet centrifugado por $2 \mathrm{~min}$ em $500 \mu \mathrm{L}$ de meio TALP (Parrish et al., 1995), suplementado com $2 \mathrm{mmol} \mathrm{L}^{-1}$ de penicilina $\mathrm{G}$ sódica, $1 \mathrm{mmol} \mathrm{L}^{-1} \mathrm{de}$ hipotaurina, $250 \mathrm{mmol} \mathrm{L}^{-1}$ de epinefrina e $10 \mu \mathrm{g} \mathrm{mL}^{-1}$ de heparina, a $200 \mathrm{~g}$. O sobrenadante foi retirado e o pellet resultante foi homogeneizado e utilizado para as mesmas avaliações realizadas antes da passagem pelo gradiente de Percoll.

A concentração espermática foi determinada no hematocitômetro, em diluição de 1:200, tendo sido avaliada tanto antes quanto após a passagem pelo gradiente de Percoll. Para a análise estatística das avaliações espermáticas antes e após o gradiente de Percoll, foi utilizado o modelo linear da análise de variância para medidas repetidas, em que foram considerados os efeitos de touro, de tratamento e da interação entre ambos como fixos, pelo GLIMMIX, SAS (SAS Institute, 2002). As comparações estatísticas foram realizadas sobre as médias ajustadas pelo método dos quadrados mínimos, com uma aproximação do teste de qui-quadrado.

\section{Resultados e Discussão}

As características de motilidade da célula espermática para os diferentes grupos, antes e após a seleção pelo gradiente de Percoll, são apresentadas na Tabela 1. A motilidade do sêmen não sexado foi superior à do sêmen sexado antes e após a passagem pelo gradiente de Percoll. O efeito do processo de sexagem na motilidade espermática foi relatado por outros autores (Hollinshead et al., 2004; Blondin et al., 2009). Nessa técnica de sexagem, o espermatozoide é submetido a vários procedimentos que podem causar danos à célula, tais como exposição ao corante e ao laser, grande pressão na passagem pelo citômetro, queda em grande velocidade dentro do tubo de colheita e permanência durante algumas horas em temperatura ambiente antes de ser processado (Chastant-Maillard \& Druat, 2005). Portanto, a queda na motilidade observada no sêmen sexado pode ter sido causada pela exposição, durante o processo de sexagem, ao corante Hoechst 33342, que é tóxico, como já foi demonstrado em espermatozoide humano (Watkins et al., 1996) e bovino (Hollinshead et al., 2004). Além disso, o efeito da exposição ao corante e, posteriormente, ao laser pode alterar a atividade mitocondrial, como foi demonstrado em trabalho realizado por Smith (1993) com ovócitos corados com Hoechst 33342 e submetidos à pequena irradiação de luz ultravioleta. Esse tipo de dano também poderia explicar a queda de motilidade observada nos espermatozoides sexados, uma vez que a função das mitocôndrias é produzir ATP para o movimento das células espermáticas (Manella, 2000).

A motilidade é uma importante variável na fecundação, sendo que diversos estudos têm sido feitos na tentativa de correlacionar motilidade e fertilidade (Correa et al., 1997; Farrell et al., 1998; Januskaukas et al., 2003; Gillan et al., 2008). É importante dizer que, na análise subjetiva, o nível de correlação pode variar entre 0,15 e 0,83 (Januskaukas et al., 2003) e a estimativa pela análise computadorizada, entre $0,82 \mathrm{e}$ 0,98 (Farrell et al., 1998). Essas diferenças observadas na correlação entre avaliação subjetiva e fertilidade podem estar relacionadas com a grande subjetividade dessa técnica em razão da variação na análise realizada por diferentes avaliadores, em diferentes momentos (Malmgren, 1997), o que pode gerar resultados equivocados ou mesmo conclusões conflitantes (Rijsselaere et al., 2003). Na avaliação subjetiva, há uma tendência em se superestimar a percentagem de espermatozoides móveis, especialmente em amostras com alta concentração espermática. Já amostras com baixa concentração, como as de sêmen sexado, podem ter suas avaliações subestimadas. No presente estudo, não foi observada diferença entre a avaliação subjetiva e a do sistema CASA (Tabela 1).

No entanto, a motilidade subjetiva tende a negligenciar a existência de subpopulações ou de outras variáveis de movimento espermático, o que pode interferir na avaliação mais exata da amostra de sêmen (Quintero-Moreno et al., 2003). Dessa forma, o conjunto dos parâmetros fornecidos pelo CASA

Tabela 1. Valores (média dos quadrados mínimos $\pm \mathrm{EP}$ ) de motilidade total de sêmen avaliada de forma subjetiva e por sistema computadorizado de análise do movimento espermático (CASA), antes e após passagem pelo gradiente de Percoll $^{(1)}$.

\begin{tabular}{lcclcc}
\hline Grupo & \multicolumn{2}{c}{ Motilidade antes do Percoll } & & \multicolumn{2}{c}{ Motilidade após Percoll } \\
\cline { 2 - 3 } \cline { 5 - 6 } & Subjetiva & CASA & & Subjetiva & CASA \\
\hline Não sexado & $63,7 \pm 1,8 \mathrm{a}$ & $58,0 \pm 3,0 \mathrm{a}$ & & $72,9 \pm 3,0 \mathrm{a}$ & $70,4 \pm 2,9 \mathrm{a}$ \\
Sexado X & $27,5 \pm 2,3 \mathrm{~b}$ & $29,6 \pm 1,3 \mathrm{~b}$ & & $39,2 \pm 2,6 \mathrm{~b}$ & $37,1 \pm 4.0 \mathrm{~b}$ \\
Sexado Y & $28,7 \pm 2,0 \mathrm{~b}$ & $26,2 \pm 2,1 \mathrm{~b}$ & & $45,0 \pm 3,0 \mathrm{~b}$ & $43,4 \pm 3,2 \mathrm{~b}$ \\
\hline (1) Médias seguidas de letras iguais, nas colunas ou nas linhas, não diferem
\end{tabular}


possibilita melhor avaliação da qualidade do sêmen (Moses et al., 1995).

$\mathrm{Na}$ Tabelas 2, são apresentadas as variáveis de movimento espermático fornecidas pelo sistema CASA para os diferentes grupos, antes e após a seleção pelo gradiente de Percoll. Nenhuma diferença foi observada entre os grupos SX e SY para todas as variáveis estudadas. Após o descongelamento, variáveis como BCF e LIN foram menores no grupo NS do que no sexado. O sêmen que continha espermatozoide sexado Y apresentou VSL semelhante ao dos grupos NS e SX, e o do grupo SX foi superior ao do grupo NS. Quanto ao VCL, todos os grupos apresentaram valores semelhantes. No entanto, após passagem pelo gradiente de Percoll, o grupo NS apresentou maiores valores de VSL e LIN do que os grupos SX e SY.

De acordo com Johnson et al. (1989), o cromossomo $\mathrm{X}$ é maior que o cromossomo $\mathrm{Y}$, e essa diferença de massa poderia influenciar o movimento espermático (Penfold et al., 1998). Porém, no presente estudo, assim como no de Penfold et al. (1998), não houve diferença no padrão de movimento dos espermatozoides que continham cromossomo $\mathrm{X}$ ou Y (Tabela 2). No entanto, a diferença encontrada na maioria dos parâmetros de cinética espermática fornecidos pelo sistema CASA entre sêmen sexado e não sexado, antes ou após a passagem pelo gradiente de Percoll, sugere que o processo de sexagem afeta não só a motilidade, mas também outros parâmetros de cinética espermática.

Para algumas dessas diferenças, os valores do grupo não sexado foram inferiores aos dos grupos sexado X e Y (Tabela 2). Esses resultados inferiores podem ser decorrentes da diferença na concentração espermática das amostras, pois o sêmen não sexado apresentou uma concentração média muito maior (35 $\pm 2,7 \times 10^{6}$ espermatozoides por dose) do que o sêmen com espermatozoides sexados $X\left(2,4 \pm 0,8 \times 10^{6}\right.$ espermatozoides por dose) e Y $\left(2,2 \pm 0,4 \times 10^{6}\right.$ espermatozoides por dose). Uma grande influência da concentração espermática nos resultados fornecidos pelo sistema CASA foi relatada por Verstegen et al. (2002). Esses autores ressaltam que, em alta concentração, as células com movimento rápido poderiam ser excluídas da análise em razão das colisões entre elas. Além da concentração, outro fator que pode alterar o padrão de movimentação da célula espermática é a viscosidade dos meios (Mortimer, 1997), pois um aumento na concentração ou na viscosidade do meio reduziria a amplitude do movimento. No presente estudo, o meio utilizado foi o mesmo para todos os grupos, portanto é possível que as diferenças encontradas para parâmetros como LIN, VCL e BCF entre sêmen sexado e não sexado resultem da menor concentração do sêmen sexado. $\mathrm{O}$ mesmo efeito da concentração não foi observado após passagem pelo gradiente de Percoll, pois, para essa avaliação, o sêmen é lavado e ressuspendido, o que diminui a viscosidade do meio e, principalmente, a diferença de concentração entre as amostras.

$\mathrm{O}$ efeito da passagem pelo gradiente de Percoll nas características estudadas para cada um dos grupos também foi avaliado. O efeito mais marcante $(p<0,05)$ foi observado na motilidade espermática: ocorreu um aumento na percentagem de espermatozoides móveis após passagem pelo Percoll nos grupos NS (Tabela 1). $\mathrm{O}$ aumento da percentagem de espermatozoides móveis em amostra de sêmen após a seleção pelo gradiente de Percoll foi relatado por vários autores (Parrish et al., 1995; Tangue et al., 2002; Trentalance \& Beorlegui, 2002; Machado et al., 2009).

Tabela 2. Valores (média dos quadrados mínimos $\pm \mathrm{EP}$ ) dos parâmetros de cinética espermática fornecidos pelo sistema CASA para sêmen antes e após a passagem pelo gradiente de Percoll ${ }^{(1)}$.

\begin{tabular}{lccccc}
\hline Grupo & VAP $\left(\mu \mathrm{m} \mathrm{s}^{-1}\right)$ & VSL $\left(\mu \mathrm{m} \mathrm{s}^{-1}\right)$ & VCL $\left(\mu \mathrm{m} \mathrm{s}^{-1}\right)$ & ALH $(\mu \mathrm{m})$ & BCF $(\mathrm{Hz})$ \\
\hline & & & Antes da passagem pelo gradiente de Percoll $(\%)$ & \\
Não sexado & $45,7 \pm 1,1$ & $30,3 \pm 1,0 \mathrm{a}$ & $78,3 \pm 0,2$ & $5,7 \pm 0,8$ & $23,3 \pm 0,9 \mathrm{a}$ \\
Sexado X & $44,9 \pm 1,2$ & $35,0 \pm 1,0 \mathrm{~b}$ & $79,0 \pm 0,2$ & $5,6 \pm 0,8$ & $28,5 \pm 0,9 \mathrm{~b}$ \\
Sexado Y & $41,9 \pm 1,3$ & $32,8 \pm 1,0 \mathrm{ab}$ & $77,1 \pm 0,2$ & $5,6 \pm 0,8$ & $27,5 \pm 0,9 \mathrm{~b}$ \\
\hline & & Após a passagem pelo gradiente de Percoll & $44,3 \pm 1,4 \mathrm{~b}$ \\
Não sexado & $51,5 \pm 1,1$ & $59,5 \pm 1,0 \mathrm{a}$ & $118,3 \pm 0,2 \mathrm{a}$ & $4,4 \pm 0,8$ & $33,3 \pm 0,9 \mathrm{ab}$ \\
Sexado X & $50,8 \pm 1,2$ & $41,1 \pm 1,0 \mathrm{~b}$ & $91,3 \pm 0,2 \mathrm{~b}$ & $4,5 \pm 0,8$ & $32,1 \pm 0,9 \mathrm{bc}$ \\
Sexado Y & $51,3 \pm 1,3$ & $43,8 \pm 1,0 \mathrm{~b}$ & $103,8 \pm 0,2 \mathrm{ab}$ & $4,6 \pm 0,8$ & $45,3 \pm 1,4 \mathrm{a}$ \\
\hline
\end{tabular}

${ }^{(1)}$ Médias seguidas de letras iguais nas colunas não diferem, pelo teste t a $5 \%$ de probabilidade $(\mathrm{N}=12)$. VAP, velocidade de trajeto; VSL, velocidade retilínea; VCL, velocidade curvilínea; ALH, amplitude lateral de cabeça; BCF, frequência de batimentos; LIN, linearidade. 
Nos parâmetros de cinética espermática, o efeito da passagem pelo gradiente de Percoll foi observado principalmente no sêmen não sexado, que apresentou aumento $(\mathrm{p}<0,05)$ nos valores de VAP, BCF e LIN, após a seleção (Tabela 2). Para as amostras de sêmen sexado $\mathrm{X}$ ou Y, a passagem pelo gradiente de Percoll afetou $(\mathrm{p}<0,05)$ os parâmetros VAP e VCL, respectivamente (Tabela 2). Essa variação na resposta entre as amostras pode ser resultante da grande diferença na percentagem de células móveis entre sêmen sexado e não sexado antes da passagem pelo gradiente de Percoll. Esses resultados sugerem que parâmetros mais específicos do movimento espermático, como os avaliados pelo sistema CASA, também podem ser afetados pela qualidade do sêmen, pois as amostras de sêmen sexado e não sexado responderam de forma diferente a um mesmo gradiente de Percoll.

Pode-se supor que alterações no protocolo de seleção, tais como concentração do gradiente de Percoll, tempo e força de centrifugação, podem mudar o resultado desse procedimento e permitir a obtenção de subpopulações com diferentes padrões de motilidade. Portanto, são necessários estudos que determinem o protocolo mais adequado para seleção espermática quando o sêmen sexado é utilizado.

Segundo Sánchez-Partida et al. (1999), assim como a motilidade, os parâmetros analisados pelo sistema CASA também têm sido correlacionados com taxas de fertilidade in vitro ou in vivo. Para condições in vitro em bovinos, é possível que a redução da motilidade e as alterações nos parâmetros de cinética espermática, fornecidos pelo sistema CASA em sêmen sexado, não sejam tão cruciais, já que nesse caso os espermatozoides são colocados diretamente em contato com o ovócito. Em contraste, para as condições in vivo, a cinética de movimento espermático é reconhecidamente um importante parâmetro na avaliação do sêmen, pois é um dos responsáveis pelo transporte do espermatozoide ao longo do trato genital (Verstegen et al., 2002), e os parâmetros de VAP e VCL são importantes para o transporte dos espermatozoides no trato reprodutivo da fêmea (Robayo et al., 2008). Portanto, apesar do seu alto custo, o sistema CASA oferece objetividade e repetibilidade nas avaliações, o que possibilita detalhar melhor a qualidade do sêmen analisado. Isso porque esse tipo de análise fornece informações adicionais sobre as características da cinética de movimento espermático não detectadas na observação visual.

\section{Conclusões}

1. O processo de sexagem por citometria de fluxo afeta a motilidade e algumas características do movimento espermático avaliadas pelo sistema computadorizado CASA.

2. A passagem pelo gradiente de Percoll aumenta a percentagem de células móveis na amostra de sêmen sexado e não sexado.

3. Sêmen sexado e não sexado apresentam resposta diferente para características do movimento espermático, quando submetidos ao mesmo protocolo de seleção por gradiente de Percoll.

\section{Agradecimentos}

À Empresa Brasileira de Pesquisa Agropecuária, pelo apoio financeiro.

\section{Referências}

ALMEIDA, G.P.; ALVAREZ, R.H. Métodos de separação de espermatozóides para escolha do sexo dos animais domésticos. Boletim da Indústria Animal, v.60, p.107-115, 2003.

ANDERSSON, M.; TAPONEN, J.; KOMMERI, M.; DAHLBOM, M. Pregnancy rates in lactating Holstein-Friesian cows after artificial insemination with sexed sperm. Reproduction in Domestic Animals, v.41, p.95-97, 2006.

BERMEJO-ÁLVAREZ, P.; RIZOS, D.; RATH, D.; LONERGAN, P.; GUTIÉRREZ-ADÁN, A. Can bovine in vitro-matured oocytes selectively process X- or Y-sorted sperm differentially? Biology of Reproduction, v.79, p.594-597, 2008.

BLONDIN, P.; BEAULIEU, M.; FOURNIER, V.; MORIN, N.; CRAWFORD, L.; MADAN, P.; KING, W.A. Analysis of bovine sexed sperm for IVF from sorting to the embryo. Theriogenology, v.71, p.30-38, 2009.

BODMER, M.; JANETT, F.; HÄSSIG, M.; DEN DAAS, N.; REICHERT, P.; THUN, R. Fertility in heifers and cows after low dose insemination with sex-sorted and non-sorted sperm under field conditions. Theriogenology, v.64, p.1647-1655, 2005.

CHASTANT-MAILlARD, S.; DRUAT, X. Sexagem de espermatozóide bovino. A Hora Veterinária, v.143, p.43-48, 2005.

CORREA, J.R.; PACE, M.M.; ZAVOS, P.M. Relationships among frozen-thawed sperm characteristics assessed via the routine semen analysis, sperm functional tests and fertility of bulls in an artificial insemination program. Theriogenology, v.30, p.721-731, 1997.

COX, J.F.; ALFARO, V.; MONTENEGRO, V.; RODRIGUEZ-MARTINEZ, H. Computer-assisted analysis of sperm motion in goats and its relationship with sperm migration in cervical mucus. Theriogenology, v.66, p.860-867, 2006.

FARRELL, P.B.; PRESICCE, G.A.; BROCKETT, C.C.; FOOTE, R.H. Quantification of bull sperm characteristics measured by computer-assisted sperm analysis (CASA) and the relationship to fertility. Theriogenology, v.49, p.871-879, 1998. 
FONSECA, V.O. da; VALE FILHO, V.R.; MIES FILHO, A.; ABREU, J.J. de. Procedimentos para exame andrológico e avaliação de sêmen animal. Belo Horizonte: Colégio Brasileiro de Reprodução Animal, 1992. 79p.

GARNER, D.L. Ancillary tests of bull semen quality. Veterinary Clinics of North America, v.13, p.313-327, 1997.

GILLAN, L.; KROETSCH, T.; MAXWELL, W.M.C.; EVANS, G. Assessment of in vitro sperm characteristics in relation to fertility in dairy bulls. Animal Reproduction Science, v.103, p.201-214, 2008.

HOLLINSHEAD, F.K.; O'BRIEN, J.K.; MAXWELL, W.M.C.; EVANS, G. Assessment of in vitro sperm characteristics after flow cytometric sorting of frozen-thawed bull spermatozoa. Theriogenology, v.62, p.958-968, 2004.

JANUSKAUSKAS, A.; JOHANNISSON, A.; RODRIGUEZ-MARTINEZ, H. Subtle membrane changes in cryopreserved bull semen in relation with sperm viability, chromatin structure, and field fertility. Theriogenology, v.60, p.743-758, 2003.

JANUSKAUSKAS, A.; JOHANNISSON, A.; SODERQUIST, L.; RODRIGUEZ-MARTINEZ, H. Assessment of sperm characteristics post-thaw and response to calcium ionophore in relation to fertility in Swedish dairy AI bulls. Theriogenology, v.53, p.859-875, 2000.

JOHNSON, L.A.; FLOOK, J.P.; HAWK, H.W. Sex preselection in rabbits: live births from $\mathrm{X}$ and $\mathrm{Y}$ sperm separated by DNA and cell sorting. Biology of Reproduction, v.41, p.199-203, 1989.

MACHADO, G.M.; CARVALHO, J.O.; SIQUEIRA FILHO, E.; CAIXETA, E.S.; FRANCO, M.M.; RUMPF, R.; DODE, M.A.N. Effect of Percoll volume, duration and force of centrifugation, on in vitro production and sex ratio of bovine embryos. Theriogenology, v.71, p.1289-1297, 2009.

MALMGREN, L. Assessing the quality of raw semen: a review. Theriogenology, v.48, p.523-530, 1997.

MANELLA, C.A. Introduction: our changing views of mitochondria. Journal of Bioenergetics and Biomembranes, v.32, p.1-4, 2000.

MORTIMER, S.T. A critical review of the physiological importance and analysis of sperm movement in mammals. Human Reproduction Update, v.3, p.403-439, 1997.

MOSES, D.F.; DELAS HERAS, M.A.; VALCÁRCEL,A.; PÉREZ,L.; BALDASSARRE, $\mathrm{H}$. Use of computerized motility analyzer for the evaluation of frozen-thawed ram spermatozoa. Andrologia, v.27, p.25-29, 1995.

PARRISH, J.J.; KROGENAES, A.; SUSKO-PARRISH, J.L. Effect of bovine sperm separation by either swim-up or Percoll method on success of in vitro fertilization and early embryonic development. Theriogenology, v.44, p.859-869, 1995.

PENFOLD, L.M.; HOLT, C.; HOLT, W.V.; WELCH, G.R.; CRAN, D.G.; JOHNSON, L.A. Comparative motility of X and Y chromosome-bearing bovine sperm separated on the basis of DNA content by flow sorting. Molecular Reproduction and Development, v.50, p.323-327, 1998 .
QUINTERO-MORENO, A.; MIRÓ, J.; TERESA RIGAU, A.; RODRÍGUEZ-GIL, J.E. Identification of sperm subpopulations with specific motility characteristics in stallion ejaculates. Theriogenology, V.59, p.1973-1990, 2003.

RIJSSELAERE, T.; VAN SOOM, A.; MAES, D.; KRUIF, A. de. Effect of technical settings on canine semen motility parameters measured by the Hamilton-Thorne analyzer. Theriogenology, v.60, p.1553-1568, 2003.

ROBAYO, I.; MONTENEGRO, V.; VALDÉS, C.; COX, J.F. CASA assessment of kinematic parameters of ram spermatozoa and their relationship to migration efficiency in ruminant cervical mucus. Reproduction in Domestic Animals, v.43, p.393-399, 2008.

SÁNCHEZ-PARTIDA, L.G.; WINDSOR, D.P.; EPPLESTON, J.; SETCHELL, B.P.; MAXWELL, W.M. Fertility and its relationship to motility characteristics of spermatozoa in ewes after cervical, transcervical, and intrauterine insemination with frozen-thawed ram semen. Journal of Andrology, v.20, p.280-288, 1999.

SARTORI, R.; SOUZA, A.H.; GUENTHER, J.N.; CARAVIELLO, D.Z.; GEIZER, L.N.; SCHENCK, J.L.; WILTBANK, M.C. Fertilization rate and embryo quality in superovulated Holstein heifers artificially inseminated with X-sorted or unsorted sperm. Animal Reproduction, v.1, p.86-90, 2004.

SAS INSTITUTE. SAS: user's guide. Versão 9.1.3. Cary: SAS Institute, 2002.

SMITH, L.C. Membrane and intracellular effects of ultraviolet irradiation with Hoechst 33342 on bovine secondary oocytes matured in vitro. Journal of Reproduction and Fertility, v.99, p.39-44, 1993.

SUAREZ, S.S.; PACEY, A.A. Sperm transport in the female reproductive tract. Human Reproduction Update, v.12, p.23-37, 2006.

TANGHE, S.; VAN SOOM, A.; STERCKX, V.; MAES, D.; KRUIF, A. de. Assessment of different sperm quality parameters to predict in vitro fertility of bulls. Reproduction in Domestic Animals, v.37, p.127-132, 2002.

TRENTALANCE, G.M.; BEORLEGUI, N.B. Sperm evaluation in cryopreserved bovine semen recovered by two selection methods. Andrologia, v.34, p.397-403, 2002.

VARNER, D.D.; VAUGHAN, S.D.; JOHNSON, L. Use of a computerized system for evaluation of equine spermatozoal motility. American Journal of Veterinary Research, v.52, p.224-230, 1991.

VERSTEGEN, J.; IGUER-OUADA, M.; ONCLIN, K. Computer assisted semen analyzers in andrology research and veterinary practice. Theriogenology, v.57, p.149-179, 2002.

WATKINS, A.-M.; CHAN, P.J.; KALUGDAN, T.H.; PATTON, W.C.; JACOBSON, J.D.; KING, A. Analysis of the flow cytometer stain Hoechst 33342 on human spermatozoa. Molecular Human Reproduction, v.2, p.709-712, 1996.

Recebido em 10 de junho de 2009 e aprovado em 9 de setembro de 2009 